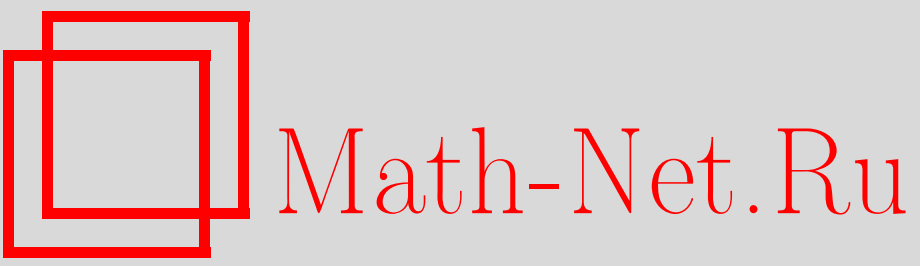

В. А. Курлин, Редукция оснащенных зацеплений к обычным, УМН, 1999, том 54, выпуск 4, 177-178

DOI: https://doi.org/10.4213/rm190

Использование Общероссийского математического портала Math-Net.Ru подразумевает, что вы прочитали и согласны с пользовательским соглашением

http://www.mathnet.ru/rus/agreement

Параметры загрузки:

IP : 34.227 .88 .159

26 апреля 2023 г., 13:22:11 


\title{
РЕДУКЦИЯ ОСНАЩЕННЫХ ЗАЦЕПЛЕНИЙ К ОБЫЧНЫМ
}

\author{
В. Курлин
}

Главный результат работы - это сведение классификации оснащенных зацеплений к классификации обычных зацеплений (теорема 1). В качестве приложения доказаны аналоги теоремы Маркова для оснащенных зацеплений и 3-многообразий. Под 3-многообразием мы понимаем ориентируемое замкнутое трехмерное многообразие. Известно, что любое 3-многообразие может быть получено перестройкой Дена из $S^{3}$ вдоль некоторого оснащенного зацепления (см. [1]). Оснащенные зацепления, полученные друг из друга преобразованиями Фенна-Рурка, задают гомеоморфные 3-многообразия (см. [2]). По аналогии с обычными зацеплениями оснащенное зацепление есть замыкание некоторой оснащенной косы, определенной с точностью до сопряжения и преобразований Маркова (теорема 3). Группа оснащенных кос $F B_{n}$ канонически изоморфна группе $B_{n} \lambda \mathbb{Z}^{n}$, где $B_{n}$ - группа (обычных) кос (теорема 2). Таким образом, любая оснащенная коса задает 3-многообразие, причем 3-многообразия гомеоморфны, если и только если косы получаются друг из друга сопряжением и преобразованиями Маркова и Фенна-Рурка. В теореме 4 геометрические преобразования Фенна-Рурка переведены на комбинаторный язык оснащенных кос. Автор благодарен Л. А. Алании за ряд ценных замечаний и В. М. Бухштаберу за редакторскую работу и поддержку.

ОПРЕДЕЛЕНИЕ 1. Оснащенное $m$-компонентное защепление - это набор $m$ непересекающихся ленточек в $\mathbb{R}^{3}$, зацепленных друг за друга и скрученных (вокруг своей средней линии) на целое число оборотов. Изотопия оснащенных зацеплений - это семейство гомеоморфизмов $\phi_{t}: \mathbb{R}^{3} \mapsto \mathbb{R}^{3}$, непрерывно зависящее от параметра $t \in[0,1]$, такое, что $\phi_{0}=\mathrm{id}, \phi_{1}$ переводит одно оснашенное зацепление в другое.

Обозначим через $L_{i}$ и $F_{i}$ края $i$-й ленты. Получим неориентируемое зацепление $L_{1} \cup F_{1} \cup \cdots \cup$ $L_{m} \cup F_{m}$. Таким образом, каждому оснащенному $m$-компонентному зацеплению соответствует обычное неориентируемое $2 m$-компонентное зацепление.

Теорема 1. Оснащенные зацепления изотопны, если и только если изотопны соответствующие обычные зацепления.

ОПРЕДЕЛЕнИЕ 2. Оснащенная коса из $n$ нитей - это набор из $n$ лент в $\mathbb{R}^{3}$ такой, что

(2.1) нижний (верхний) край $i$-й ленты - это отрезок $[(2 i, 0,0),(2 i+1,0,0)] \subset \mathbb{R}^{3}([(2 i, 0,1)$, $(2 i+1,0,1)] \subset \mathbb{R}^{3}$, соответственно);

(2.2) каждая лента монотонно поднимается от нижнего края к верхнему;

(2.3) каждая лента может быть скручена целое число раз.

Рассматривая вместо лент их края, получим обычную косу из $2 n$ нитей. Множество $F B_{n}$ оснащенных кос наследует естественную групповую структуру из обычных кос и является подгруппой в группе $B_{2 n}$. Существует естественное действие перестановок $s \in S_{n}$ на

$$
\mathbb{Z}^{n}=\left\{\bar{f}=\left(f_{1}, \ldots, f_{n}\right), f_{i} \in \mathbb{Z}\right\}: s\left(f_{1}, \ldots, f_{n}\right)=\left(f_{s(1)}, \ldots, f_{s(n)}\right) .
$$

Определим полупрямое произведение $B_{n} \lambda \mathbb{Z}^{n}$ как можество пар $(\alpha, \bar{f})$ с групповой операцией (здесь $s=s(\beta)^{-1}$ )

$$
(\alpha, \bar{f}) \circ(\beta, \bar{g})=(\alpha \beta, s(\bar{f})+\bar{g}) .
$$

Зададим отображение $\Phi: B_{n} 入 \mathbb{Z}^{n} \rightarrow F B_{n}$ формулой

$$
\Phi(\sigma, \bar{f})=\Delta(\sigma) \tau_{1}^{2 f_{1}} \cdots \tau_{2 n-1}^{2 f_{n}},
$$

где $\tau_{i}$ - образующие группы $B_{2 n} \supset F B_{n}$ и $\Delta: B_{n} \rightarrow B_{2 n}$ - раздваивающий гомоморфизм, определенный на образующих $\sigma_{i}$ по формуле $\Delta\left(\sigma_{i}\right)=\tau_{2 i} \tau_{2 i-1} \tau_{2 i+1} \tau_{2 i}$. 
ТеОрема 2. Ф является изоморфизмом.

Простым следствием теоремы Маркова (см. [3]) о зацеплениях и теоремы 1 является следующая

Теорема 3. Оснащенные косы при замыкании дают изотопные оснащенные зацепления, если и только если одна получается из другой конечной последовательностью следующих преобразований:

(1) сопряжение $\alpha \mapsto \beta \alpha \beta^{-1}, \alpha, \beta \in B_{n}$;

(2) преобразования Маркова $\alpha \leftrightarrow \alpha \sigma_{n}^{ \pm 1}, \alpha \in B_{n} \subset B_{n+1}$.

Любое оснащенное зацепление можно расположить на плоскости (см. [4]) так, что каждая лента почти полностью лежит на плоскости, лишь несколько раз поднимаясь над ней, когда зацепляется с другой лентой или скручивается вокруг себя, образуя при этом петельки.

ОПРЕДЕЛЕнИЕ 3. Преобразования Фенна-Рурка оснащенных зацеплений, расположенных на плоскости, заключаются в добавлении и удалении \pm 1 -оснащенной незаузленной ленты. При этом ленты, которые проходят сквозь нее (их можно расположить параллельно рядом друг с другом), как единое целое скручиваются в виде большой петли. Такую петлю можно нарисовать двумя способами. Нужньй способ выбирается в соответствии с оснащением добавленной (или удаленной) ленты. В частности, можно добавлять и удалять \pm 1 -оснашенную ленту, расположенную вдалеке от остальной части зацепления.

ТЕорема 4. Оснащенные косы задают гомеоморфнье 3-многообразия, если и только если одна получается из другой конечной последовательностью следующих преобразований

(1) сопряжсение $\alpha \mapsto \beta \alpha \beta^{-1}, \alpha, \beta \in B_{n}$;

(2) преобразования Маркова $\alpha \leftrightarrow \alpha \sigma_{n}^{ \pm 1}, \alpha \in B_{n} \subset B_{n+1}$;

(3) преобразования Фенна-Рурка $\sigma \leftrightarrow \sigma f r_{n}^{ \pm}(i, j), \sigma \in F B_{n} \subset F B_{n+1}$, где мы обозначили

$$
\begin{gathered}
f r^{+}(i, j)=\Phi\left(\sigma^{+}(i, j), n \text {-кортежс }(1, \ldots, 1)\right), \\
f r^{-}(i, j)=\Phi\left(\sigma^{-}(i, j), n-\operatorname{kopmexc}(-1, \ldots,-1)\right), \\
\sigma^{+}(i, j)=\alpha\left(\sigma_{n}^{2}\right)\left(\sigma_{n-1} \sigma_{n}^{2} \sigma_{n-1}\right) \cdots\left(\sigma_{n-i-j+2} \cdots \sigma_{n}^{2} \cdots \sigma_{n-i-j+2}\right) \beta, \\
\sigma^{-}(i, j)=\alpha\left(\sigma_{n}^{-2}\right)\left(\sigma_{n-1} \sigma_{n}^{2} \sigma_{n-1}\right) \cdots\left(\sigma_{n-i-j+2}^{-1} \cdots \sigma_{n}^{-2} \cdots \sigma_{n-i-j+2}^{-1}\right) \beta, \\
\alpha=\left(\sigma_{n} \cdots \sigma_{n-j+1}\right)\left(\sigma_{n-j}^{-1} \cdots \sigma_{n-i-j+1}^{-1}\right), \quad \beta=\left(\sigma_{n-i-j+1}^{-1} \cdots \sigma_{n-j}^{-1}\right)\left(\sigma_{n-j+1} \cdots \sigma_{n}\right) .
\end{gathered}
$$

Заметим, что преобразования Маркова выводят косу из подгруппы $F B_{n}$ в группу всех кос $B_{2 n}$. Преобразования Фенна-Рурка могут применяться только к оснащенным косам из $F B_{n}$.

\section{СПИСОК ЛИТЕРАТУРЫ}

[1] Матвеев С. В., Фоменко А. Т. Алгоритмические и компьютерные методы в трехмерной топологии. М.: Изд-во МГУ, 1991. [2] Lu N. // Trans. Amer. Math. Soc. 1992. V. 331. P. 143-156. [3] Morton H. // Math. Proc. Cambridge Philos. Soc. 1986. V. 99. P. 247-260. [4] Прасолов В. В., Сосинский А. Б. Узлы, зацепления, косы и трехмерные многообразия. М.: МЦНМО, 1997.

Московский государственный 